\title{
Kartenportal.CH: zehn Jahre Erfahrung mit einem innovativen Suchinstrument
}

\begin{abstract}
Kartenportal.CH wurde vor zehn Jahren gestartet, um die Katalogsuche nach Karten zu vereinfachen und um die dezentral verteilten Kartenbestände in der Schweiz virtuell zusammenzuführen. Der Aufbau des nationalen Wissenschaftsportals e-lib.ch bot die zu Beginn notwendige Startfinanzierung und wichtige Symbiosen mit Partnerprojekten. Mittlerweile ist Kartenportal.CH ein in unterschiedlichen Nutzerkreisen etabliertes Instrument für die Suche nach Karten in Schweizer Bibliotheken. Wegen seiner innovativen Technologie und den spezifischen Lösungsansätzen für den Nachweis von umfangreichen und auf viele Institutionen verteilten Kartenbeständen ist Kartenportal.CH auch ausserhalb der Schweiz mindestens in Fachkreisen bekannt. Acht Partnerinstitutionen sorgen mit einer soliden Finanzierung für einen nachhaltigen Betrieb und den Ausbau des Angebots. Metadaten und teilweise auch Vorschaubilder zu rund 350’000 Karten können über eine räumliche Suche gefunden werden.
\end{abstract}

Kartenportal.CH bietet eine etablierte und in Fachkreisen auch über die Landesgrenzen hinaus bekannte Suchmaschine für Karten in Schweizer Bibliotheken (www.kartenportal.ch). Das für die Öffentlichkeit kostenfreie Online-Angebot wurzelt in einem vor bald einem Jahrzehnt formulierten Konzept, für dessen Realisierung das technische Instrumentarium (besonders die Geosuche) noch nicht vorhanden war. Die Rückschau überfliegt Erinnerungen an Enthusiasmus, Ideale, Gottvertrauen und Visionen - aber auch an Sackgassen, Frustrationstoleranz und kalkulierten Wagemut. Dass Kartenportal.CH heute erfolgreich funktioniert und auf zahlreiche Berichterstattungen in öffentlichen Medien verweisen kann, ist einerseits vielleicht ein paar glücklichen Zufällen $\mathrm{zu}$ verdanken. Aber ohne den Gestaltungs- und Durchhaltewillen verschiedener Fachkräfte aus unterschiedlichen Schweizer Bibliotheken hätten diese Zeilen nicht geschrieben werden können. Von Anfang an forderte das Projekt bibliothekarische und fachspezifische Erfahrung, konstruktive Zusammenarbeit und eine stetige Weiterbildung seiner treibenden Kräfte - vor allem in der Informationstechnologie.

Als die kantonalen Universitätsbibliotheken zusammenfanden, um 2008 mit dem Projekt e-lib.ch den Aufbau eines nationalen Wissenschaftsportals zur Elektronischen Bibliothek Schweiz in Angriff zu nehmen, wurde die schweizerische Arbeitsgruppe für Karten des bibliothekarischen Berufsverbandes aufmerksam. Schon seit einigen Jahren diskutierte sie Möglichkeiten, wie Karten in Bibliotheks- 
beständen über öffentlich zugängliche Online-Kataloge (OPACs) von Benutzerinnen und Benutzern besser gefunden werden können. Denn die Suche nach geographischen Karten in konventionellen Bibliothekskatalogen war schwierig, zeitintensiv und erforderte meistens Spezialwissen. Wenn bibliographische Angaben wie Titel oder Autor nicht im Voraus bekannt waren, führten textbasierte Recherchen bei diesen Dokumenttyp selten zum Ziel. Auch die damals noch neuen Facettierungsmöglichkeiten in den OPACs, bei denen Kartenmaterialien aus Trefferlisten herausgefiltert werden können, behoben das Problem nur in ausgewählten Fällen. Erschwerend kam in der Schweiz die föderale und dezentrale Bibliothekslandschaft hinzu, in der viele spezialisierte Kartensammlungen über das Land verteilt sind.

Die Idee einer räumlich-graphischen Suche im elektronischen Katalog wurde bereits 2004 von Jürg Bühler (ETH-Bibliothek) skizziert. ${ }^{1}$ Als Präsident der oben genannten Arbeitsgruppe überzeugte er deren Mitglieder schnell davon, einen gemeinsamen Antrag zuhanden des nationalen Projektes e-lib.ch einzureichen, nachdem die Schweizer Bibliothekare schon seit fast drei Jahrzehnten Koordinaten erfassten, die noch nicht ausgewertet werden konnten. Drei Exponenten dieser Fachgruppe (aus der ETH-Bibliothek, der Forschungsanstalt WSL und der Zentralbibliothek Zürich) formulierten schliesslich den Plan zur Realisierung einer virtuellen geographischen Bibliothek mit einer räumlichen Suche in einem aus verschiedenen Verbünden bestehenden nationalen Katalog. Noch war nicht klar, ob sich diese Vision als Utopie entpuppen würde, da ähnliche Ansätze in der Bibliotheks- und Archivwelt noch nicht realisiert waren. Deshalb regierte im August 2008 die Vorsicht, als der Antrag für eine „Virtuelle Fachbibliothek Geoinformation“ eingereicht wurde. Das visionäre Desiderat war nur eines von insgesamt fünf Projektzielen und es war als letztes aufgelistet: „Zentraler Nachweis von Karten und Geodaten durch Zugang in Bibliothekskataloge (OPAC), Prüfung und Integration eines Tools für die neuartige räumlich-graphische Suche nach Karten und Geodaten und Entwicklung einer Datenbank für einen Gesamtkatalog.“ Die übrigen Punkte standen im Zeichen der Bemühungen, einen übersichtlichen Zugriff auf online verfügbare Geodaten in Bibliotheken und anderen Informationsstellen zu ermöglichen. Gemeint war damit vor allem die Katalogisierung von Links zu digitalen Kartenangeboten. Die Entwicklung eines solchen Metadatenkatalogs und die Erfassung beziehungsweise Beschreibung von rund 300 Links zu Online-Karten gehörten zu den ersten Meilensteinen dieser virtuellen

1 Jürg Bühler: Kartenkataloge der Zukunft. Die graphische räumliche Suche. In: Ders. (Hrsg.): Die digitale Kartenbibliothek - eine Momentaufnahme. München 2004 (Kartensammlung und Kartendokumentation, Beiheft 1), S. 215-222. 
Fachbibliothek. Informationen zu Karten in Bibliotheksbeständen, die über den Metadatenkatalog nicht erschlossen werden konnten, wurden über ein Verzeichnis aller öffentlichen Schweizer Kartensammlungen mit ihren OPAC-Verbindungen zur Verfügung gestellt (Abb. 1). Die entsprechende Gewichtung äusserte sich bald in der neuen Projektbezeichnung „Kartenportal.CH“.

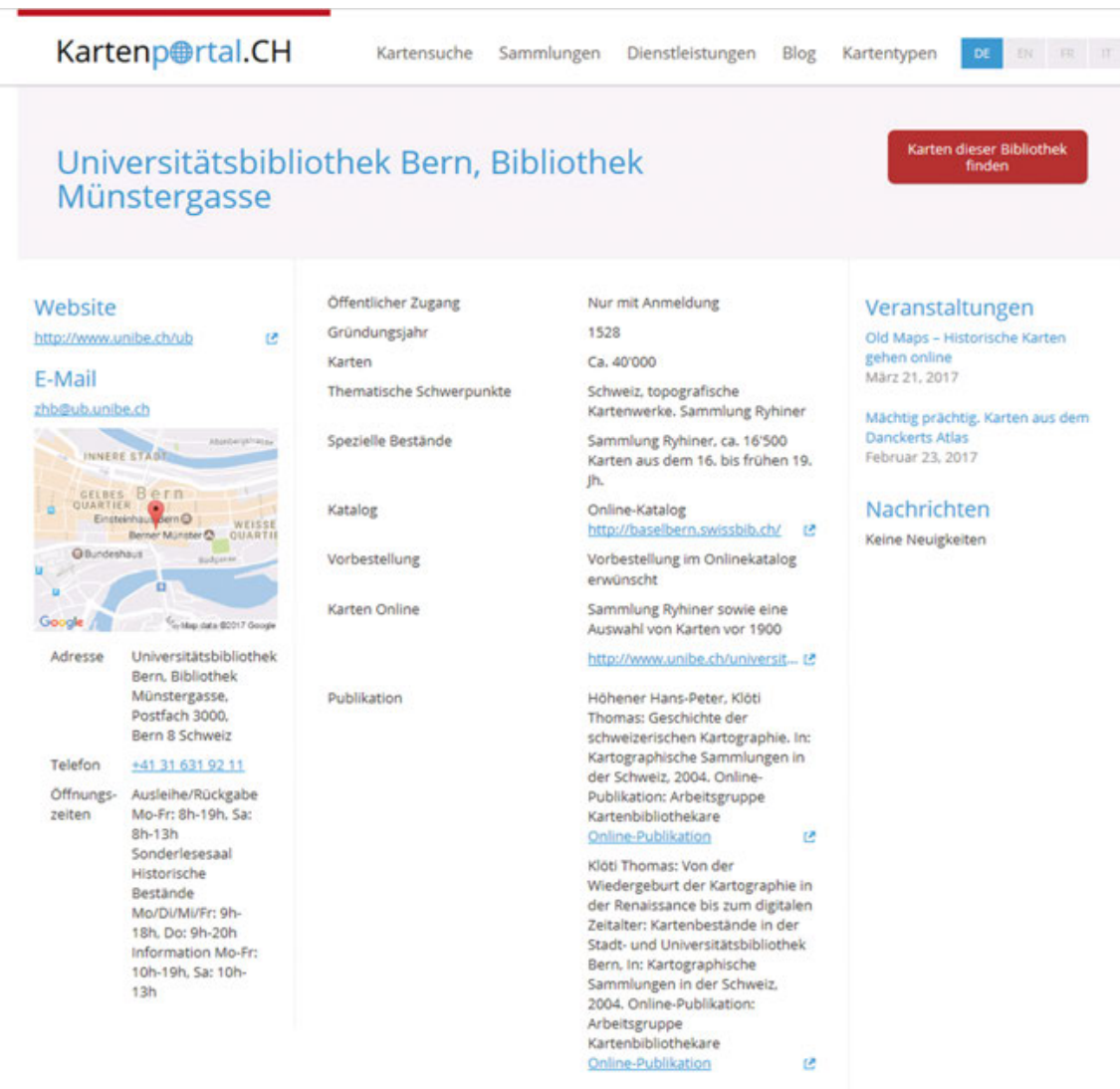

Abb. 1: alphabetisch, stichwortartig und räumlich-graphisch suchbares Sammlungsverzeichnis mit Hinweisen zum Bestand, zur Benutzung und zu aktuellen Veranstaltungen der angezeigten Institution

Es zeigte sich schon bald, dass Kooperationen mit anderen e-lib.ch-Projekten günstige Synergien schufen. Die Zusammenarbeit mit swissbib einerseits realisierte schon früh die geplante zentrale Abfrage nach Kartenmaterialien in Schweizer Bibliotheken. Dank der Hilfe von ElibEval andererseits wurden die Benutzer- 
freundlichkeit und die Barrierefreiheit anhand verschiedener ,Usability'-Evaluationen laufend verbessert. Der ursprüngliche Plan sah vor, bei einem Projektpartner beziehungsweise bei einer nahestehenden Institution die räumlich-geographische Suche $\mathrm{zu}$ günstigen Konditionen zeitnah entwickeln $\mathrm{zu}$ lassen. Diese Hoffnung zerschlug sich schliesslich aufgrund der fehlenden personellen Ressourcen, die ein solches Projekt fristgerecht in Angriff hätten nehmen können. Kartenportal.CH sah 2009 dem Ende seiner Projektfinanzierung entgegen. Es bot im Sinne einer Veredelung von swissbib einen zentralen Sucheinstieg für die Kartenrecherche in schweizerischen OPACs, ein Verzeichnis der kartographischen Sammlungen in der Schweiz und einen wenig bekannten und entsprechend kaum genutzten Metadatenkatalog für die Links zu Online-Karten.

Gerade als bei Kartenportal.CH die Euphorie in Nüchternheit umzuschlagen drohte, machte der Softwareingenieur Petr Přidal auf verschiedenen wissenschaftlichen Tagungen auf sein Projekt OldMapsOnline aufmerksam. ${ }^{2}$ Dieses sah den Aufbau einer räumlichen Suche von Kartenmaterial für den Katalog der Mährischen Bibliothek in Brünn vor. Nachdem sich Google Maps und Google Earth etabliert hatten und die Open Source-Bewegung die Entwicklung von webbasierten Benutzerschnittstellen vorantrieb, war eine einfache und kostengünstige Lösung in Sicht. So konnten sich die Projektpartner mit Petr Přidal trotz der knappen Restmittel auf die Entwicklung eines auf dieser Technologie basierenden Prototyps einigen. Dieser würde Kartenportal.CH das öffentliche Interesse sichern und die Nachfolgefinanzierung erleichtern. Die Bedingung an den Softwareingenieur war, eine Firma zu gründen und diese in der Schweiz anzumelden. Diese Klokan Technologies GmbH mit Sitz im Kanton Zug setzte 2010 die Entwicklung des Prototyps gemäss dem ihm vorgelegten Lastenheft rechtzeitig um. So konnte sich Kartenportal.CH 2011 erfolgreich um eine Teilnahme bei dem mittlerweile von e-lib.ch lancierten Nachfolgeprojekt bewerben (die Alternative sah die Gründung eines Fördervereins vor). Nach einer Überbrückungsfinanzierung aus Restmitteln von e-lib.ch richteten sich die Bemühungen nach 2012 in erster Linie darauf, den Prototyp der räumlichen Suche in einen funktionierenden Betrieb überzuführen. Damit bot Kartenportal.CH erstmals in der Geschichte eine räumliche Suche über grosse Datenbestände aus verschiedenen Bibliotheken beziehungsweise aus verschiedenen Verbundkatalogen. ${ }^{3}$ Zudem konnte bisher keine andere Anwendung alle Kartenmaterialien eruieren, die innerhalb eines Suchbereichs liegen, diesen anschneiden oder überlappen. Die wenigen beste-

2 Thomas Klöti, Jost Schmid: Geographische Suche nach Kartenmaterial. In: Cartographica Helvetica 43 (2011), S. 42.

3 Thomas Klöti, Jost Schmid: Suche nach gedruckten und digitalen Karten mit kartenportal.ch. In: arbido 3/2011, S. 27-30. 
henden Lösungen für die räumliche Suche funktionierten nur in verhältnismässig kleinen Sonderbeständen und sie berücksichtigten keine Flächenfunktionen, sondern nur Zentrums- oder Eckpunkte von Karten - entsprechend schwierig war die Interpretation der angezeigten Treffer. Die Recherche in der online verfügbaren räumlichen Suche von Kartenportal.CH erfolgt intuitiv und sie erfordert im Gegensatz zum herkömmlichen Katalog kein Expertenwissen. Darüber hinaus werden die Perimeter der in der Trefferliste angewählten Dokumente in der Suchkarte rot markiert, was die Interpretation der Antwort aus dem Recherchetool erheblich vereinfacht. Die Navigation in der Suchkarte kennen die Nutzerinnen und Nutzer von Google Maps, da diese Anwendung zusammen mit dem Ortsverzeichnis über eine Programmierschnittstelle in Google Maps eingebunden ist.

Entsprechend dem Zoomfaktor und den gewählten Attributen wird in der Kartensuche - analog zu den gängigen Suchmaschinen - sofort eine gewichtete Trefferliste angezeigt. Für Vorgaben, die die Entstehungszeit der gewünschten Karte betreffen, kann eine Zeitschiene entsprechend eingestellt werden. Die Suche kann auf bestimmte Bibliotheken beschränkt werden. Eine zusätzliche Filtermöglichkeit besteht in der Wahl eines grossen, mittleren oder kleinen Massstabs. Eine erweiterte Suchmöglichkeit erlaubt mittlerweile die Eingabe von Expertenwissen wie Angaben zum Autor, Verlag, Thema und Titel, damit die Treffermenge noch konziser wird. Diese wird in einer Spalte rechts in der räumlichen Suche angezeigt. Die Reihenfolge der angezeigten Titel wird von einem neu entwickelten Ranking-Algorithmus namens „MapRank“ bestimmt. Diese Technologie berücksichtigt eine räumliche Ähnlichkeitsfunktion, die den Perimeter des Kartenmaterials mit demjenigen des durch die Suchanfrage des Benutzers abgedeckten Gebietes vergleicht. ${ }^{4}$ Das Ergebnis wird mit den Angaben aus dem Filter kombiniert. Nach dieser Berücksichtigung von Erscheinungszeitraum, Massstab und Volltext ergibt sich das endgültige Ranking. Dieses folgt dem Prinzip des Wahrscheinlichkeitsrankings, nach dem die Dokumente in absteigender Reihenfolge ihrer vermuteten Relevanzwahrscheinlichkeit für die Benutzer sortiert werden.

MapRank verwendet eine spezielle Form der Indexierung und ermöglicht so die kurzen Antwortzeiten nach der Suche in zurzeit etwa 350'000 verschiedenen Datensätzen. Die Technologie verwendet dabei einen Index, der bereits einen Teil des vorausberechneten Rankings der Dokumente enthält. Diese Vorausberechnung erfolgt täglich, wenn die Datensätze über eine OAI-PMH-Schnittstelle aus

4 Vgl. Markus Oehrli u. a.: MapRank. Geographical Search for Cartographic Materials in Libraries. In: D-Lib Magazine 17 (2011) (http://www.dlib.org/dlib/september11/oehrli/09oehrli.html). Alle Links in diesem Beitrag wurden am 26.03.2018 überprüft. 
swissbib beziehungsweise aus den einzelnen Verbundkatalogen importiert werden. Die Benutzenden zeigen sich meist überrascht, wenn sie bereits beim Start mit Treffern konfrontiert werden, obwohl noch keine bewusste Aktion ausgelöst wurde. Jede Aktion bei der Suchkarte oder bei den Attributen führt zu unmittelbaren Veränderungen bei der Trefferanzeige, was schnelle Erfolgserlebnisse garantiert. Grosse Begeisterung löst die Zeitschiene aus: Deren Bedienung wird im Vergleich zu den Filterkonzepten konventioneller OPACs als sehr komfortabel empfunden.

Aus der konventionellen Benutzung grosser Kartensammlungen wird regelmässig berichtet, dass Benutzerinnen und Benutzer Karten zu sehen wünschen, deren Metadaten sie von zuhause aus über Kartenportal.CH gefunden haben. Das Suchportal verweist naturgemäss auch auf Bestände, die aufgrund von Urheberrechtsbestimmungen nicht online visualisiert werden können und nach wie vor in einem Lesesaal konsultiert werden müssen. Durch das Attribut „nur gescannte Karten zeigen“ lässt sich die Recherche auf digitalisierte Bestände reduzieren: Bereits in der Metadatenanzeige verheisst ein Vorschaubild, dass die entsprechende Karte über einen angegebenen Link in hoher Auflösung sofort einzusehen ist (Abb. 2). Diese Verbindung führt in den meisten Fällen zu spezialisierten Visualisierungsplattformen wie zum Beispiel e-manuscripta.ch oder e-rara.ch. Bei vielen mehrblättrigen Kartenwerken kann auf Kartenportal.CH zudem über ein entsprechendes Symbol eine Übersicht aufgerufen werden, die die einzelnen Blattschnitte (d.h. die Einteilung des Kartenwerkes) auf der Suchkarte sichtbar macht.

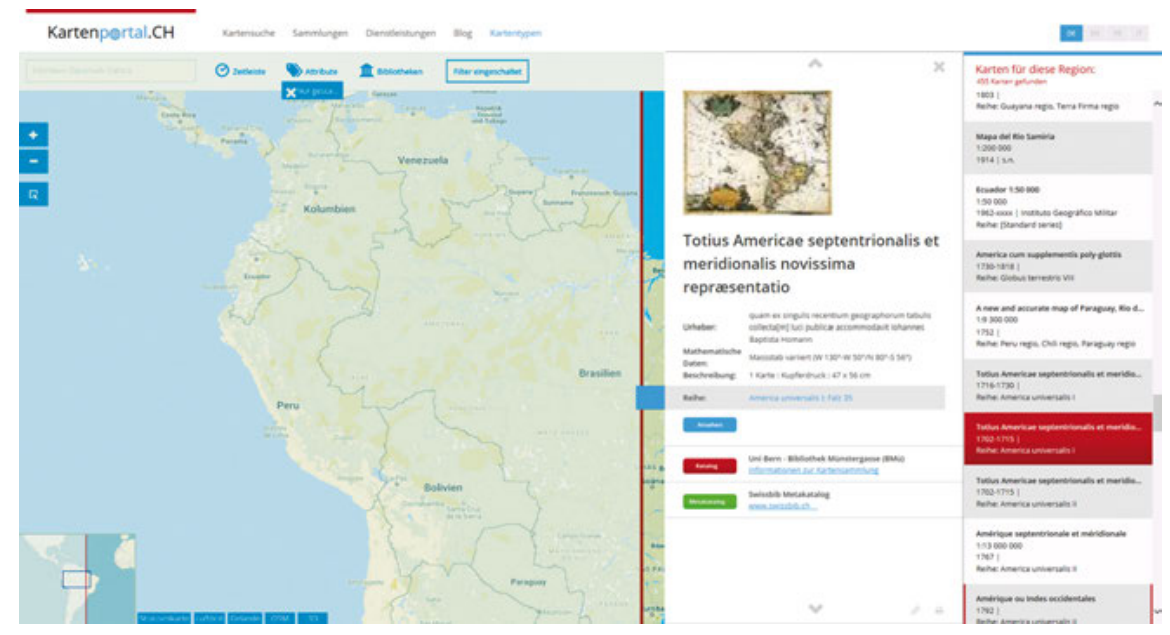

Abb. 2: Eine Filterfunktion ermöglicht die Kartensuche ausschliesslich in online visualisierten Beständen. Dank einem eigens für diese Anwendung entwickelten Ranking-Algorithmus kann rechts eine nach Relevanz gewichtete Trefferliste in Echtzeit angezeigt werden. 
Das eigentliche Erfolgsgeheimnis der räumlichen Suche mit dem MapRankAlgorithmus bei Kartenportal.CH ist die Tatsache, dass sie bei der Nutzung eines Metakatalogs mit sehr grossen Datenbeständen ihre Stärken voll ausspielen kann. Dazu gehört ihre Robustheit, die jederzeit eine effiziente Suche in Daten unterschiedlicher Herkunft ermöglicht, sowie ihre Unabhängigkeit von unterschiedlichen Sacherschliessungskonzepten, die eine konsolidierte Suchmaschine korrumpieren könnten. Die von Klokan Technologies für Kartenportal.CH entwickelte Technologie beförderte so die glückliche Symbiose dieses Geoportals mit swissbib zu einer wegweisenden Pionierleistung. Aus dem am Projekt beteiligten Kreis, der mittlerweile auf acht Partnerinstitutionen angewachsen ist, erschienen Fachartikel in wissenschaftlichen Zeitschriften $\mathrm{zu}$ den Innovationen bei Kartenportal.CH (wie in den Fussnoten angegeben). Ein breites internationales Echo fand eine Mitteilung an einschlägige Print- und E-Medien. Die räumliche Suche und die MapRank-Technologie kommen mittlerweile in verschiedenen Anwendungen weltweit zum Einsatz, was der ursprünglich aus einer Person bestehenden Entwicklerfirma massgeblich zu einem Ausbau des Mitarbeiterstabes verhalf. Entsprechend solide und nachhaltig ist dadurch der Support von Kartenportal.CH von technischer Seite her aufgestellt. Unter diesen Anwendungen befinden sich hauptsächlich Plattformen, die einer Visualisierung von urheberrechtsfreien Sammlungsbeständen dienen und explizit nur zu Dateien von alten Karten führen (www.oldmapsonline.org, www.davidrumsey.com, www. mapy.mzk.cz). Kartenportal.CH versteht sich darüber hinaus als Metakatalog mit räumlichen Suchmöglichkeiten, die auch zu urheberrechtsgeschützten Beständen führen (diese machen in den grossen Sammlungen die Mehrheit aus). Wie bereits oben erwähnte Erfahrungen zeigen, führen solche Treffer schliesslich zum Besuch von traditionellen Lesesälen, wo entsprechendes Kartenmaterial zur Benutzung vorgelegt werden kann.

Auf internationalen Symposien wird bei Projektpräsentationen immer wieder auf Kartenportal.CH als Benchmark verwiesen: Im Kartenhistorischen Colloquium 2016 in Wien zum Beispiel stellte die Österreichische Akademie der Wissenschaften ihre Erschliessungsarbeiten an der Sammlung Woldan vor. Die räumliche Suche solle nach dem Vorbild von Kartenportal.CH erfolgen. In einer anderen Präsentation wiesen Mitarbeiterinnen der Kartensammlung der Universitätsbibliothek Wien auf den bei Kartenportal.CH (dank swissbib) konsolidierten und dedoublierten nationalen Kartenkatalog hin. Diese Anwendung, insbesondere ihre Gesamtschau über gescannte Bestände, bleibt ausserhalb der Schweiz ein Desiderat zur besseren Koordinierung von Digitalisierungsprojekten. Es lässt sich im Ausland noch nicht verhindern, dass verschiedene Institutionen die gleichen Bestände mit identischen Kartenausgaben einscannen. 
Für Kartenportal.CH war die Tatsache, dass ein Verzeichnis der Schweizer Kartensammlungen mit Beschreibungen zu den einzelnen Kartenbeständen und ihrer Geschichte in der zweiten Hälfte des letzten Jahrzehnts nicht als Druck erscheinen konnte, ein weiterer Glücksfall: Die somit bereits vorliegenden Informationen zu Benutzung, Bestandsbeschreibungen und Kontaktadressen der zahlreichen Institutionen konnten auf Kartenportal.CH eingebunden und mit wenig Aufwand über eine Umfrage auf den neuesten Stand gebracht werden. Eine besondere Aufwertung erfuhr das Verzeichnis durch die Verlinkung auf bis dahin unpublizierte Aufsätze zu den wichtigsten Sammlungen. Seit wenigen Jahren ist es über 90 Sammlungsverantwortlichen über einen passwortgeschützten Zugang möglich, die eigenen Angaben laufend zu ändern beziehungsweise $\mathrm{zu}$ aktualisieren. Zudem können sie fachspezifische Anlässe und Ausstellungen auf Kartenportal.CH bewerben und in einem Blog Neuentdeckungen beschreiben (Abb. 3). Das Verzeichnis der Kartensammlungen verfügt über eine eigene Suchkarte, die eine Übersicht über die nächstgelegenen Sammlungen liefert oder deren genaue Lokalisierung ermöglicht. Eine eigene Rubrik beschreibt verschiedene Kartentypen mit kurzen Texten und anschaulicher Illustration, so dass sie auch für schulische Zwecke verwendet werden kann. Unter der Überschrift „Dienstleistungen“ bietet sich ein Tutorial an, dass auf die speziellen Herausforderungen bei der Kartenrecherche aufmerksam macht und auf die verschiedenen Möglichkeiten hinweist, die sich aus einer persönlichen Kontaktaufnahme mit qualifiziertem Fachpersonal ergibt. 


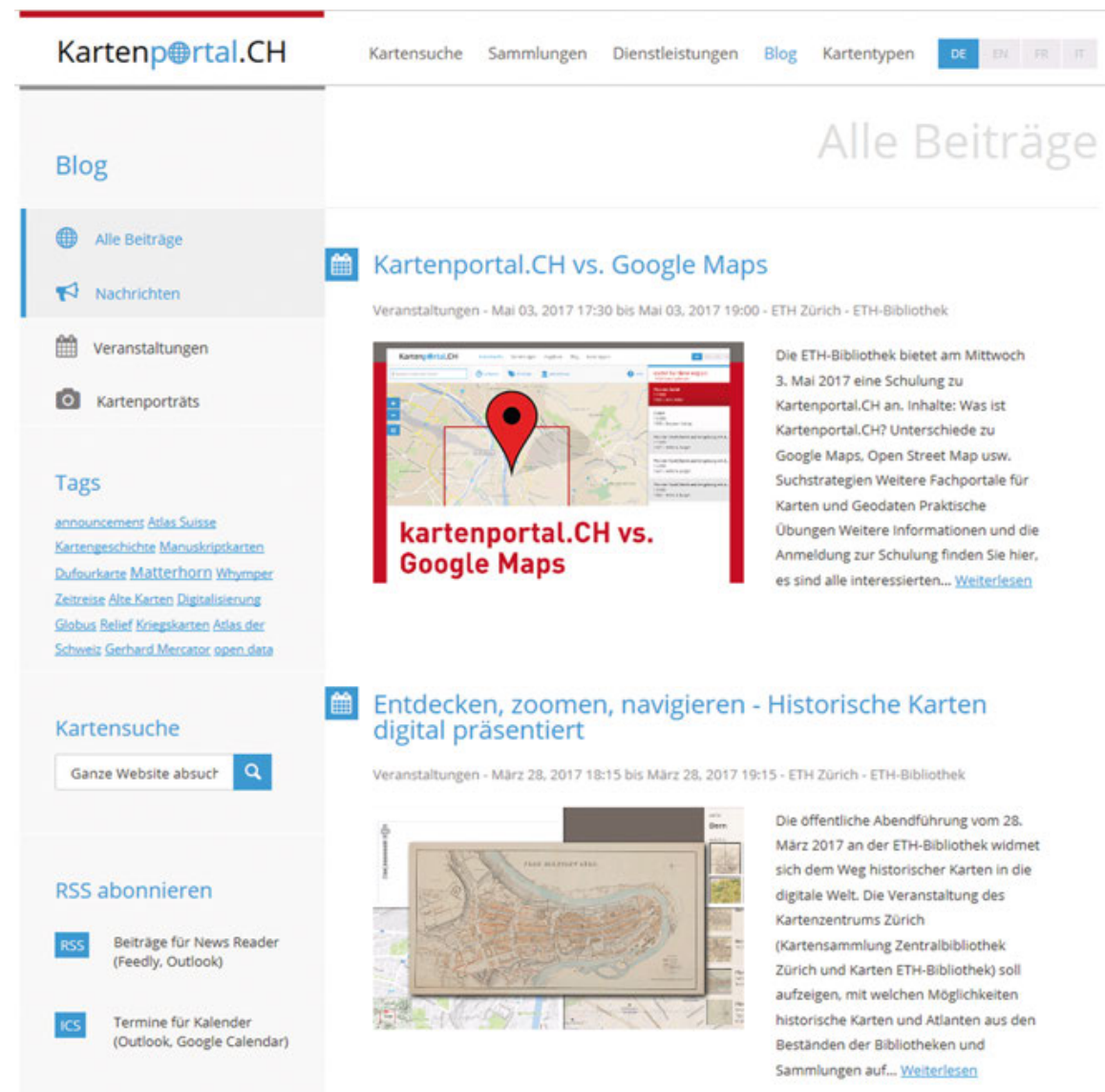

Abb. 3: Der Karten-Blog, in dem die Verantwortlichen von etwa 90 Schweizer Kartensammlungen Neuigkeiten, Entdeckungen, Historisches oder anstehende Veranstaltungen verkünden können.

Die verhältnismässig niedrigen Betriebskosten von Kartenportal.CH erleichterten 2014 die Gründung eines finanziell unabhängigen Konsortiums unter der Geschäftsleitung der Zentralbibliothek Zürich. Als ihre Vertragspartnerinnen zeichneten die Institutionen, von denen die meisten schon von Beginn an in das Projekt involviert waren; namentlich die ETH-Bibliothek, die Universitätsbibliothek Basel, die Universitätsbibliothek Bern, die Schweizerische Nationalbibliothek, die Kantonsbibliothek Vadiana St. Gallen und Lib4RI. Neu dazu gestossen ist im Gründungsjahr des Konsortiums das Bundesamt für Landestopografie swisstopo. Damit verweist die breite Finanzierungsbasis (in drei verschiedenen Beitragskategorien) nicht nur auf die allgemeine Akzeptanz von Kartenportal.CH in verschiedenen Landesteilen bei fachlich massgebenden Einrichtungen, son- 
dern sie ermöglicht auch eine im Vergleich bescheidene Beitragshöhe, die die künftige Finanzierung auf eine nachhaltige Basis stellt. Zum politischen Gleichgewicht im Konsortium trägt entscheidend bei, dass Kartenportal.CH bei einem unabhängigen (schweizerischen) Drittanbieter gehostet wird und dass jede Partnerinstitution mit einer gleichberechtigten Stimme in einem Steuerungsausschuss vertreten ist. Im selben Zug kann dieser Steuerungsausschuss auch starke Partikularinteressen berücksichtigen, sofern sich die involvierten Institutionen um die notwendige Finanzierung kümmern. So ermöglichte die ETH-Bibliothek 2016 zum Beispiel die Implementierung eines neuen Content Management Systems (CMS), um vor allem die Redaktion im Rahmen der Mehrsprachigkeit von Kartenportal.CH zu vereinfachen (Deutsch, Englisch, Französisch, Italienisch). Damit reagiert Kartenportal.CH auf ein Desiderat der bundesnahen Betriebe.

Zusätzlich zur Sicherstellung des Betriebs werden gemäss Finanzplanung jährlich Rücklagen für Anpassungen und kleinere Weiterentwicklungen gemacht. 2017 wurde zum Beispiel ein innovativer ,3D‘-Globus als optionale Suchkarte implementiert (Abb. 4). Damit ermöglicht Kartenportal.CH erstmals die Recherche von Kartenmaterialien zu den Polgegenden (bei herkömmlichen Suchkarten sind diese Regionen projektionsbedingt nicht abgebildet). Regelmässige Neuerungen wie diese stellen sicher, dass Kartenportal.CH im Gespräch bleibt und dass die seit über drei Jahren stabile Benutzung bei rund 18'000,echten Besuchen' pro Jahr auch in Zukunft die solide Nachfrage für das spezialisierte Online-Angebot sicherstellt.

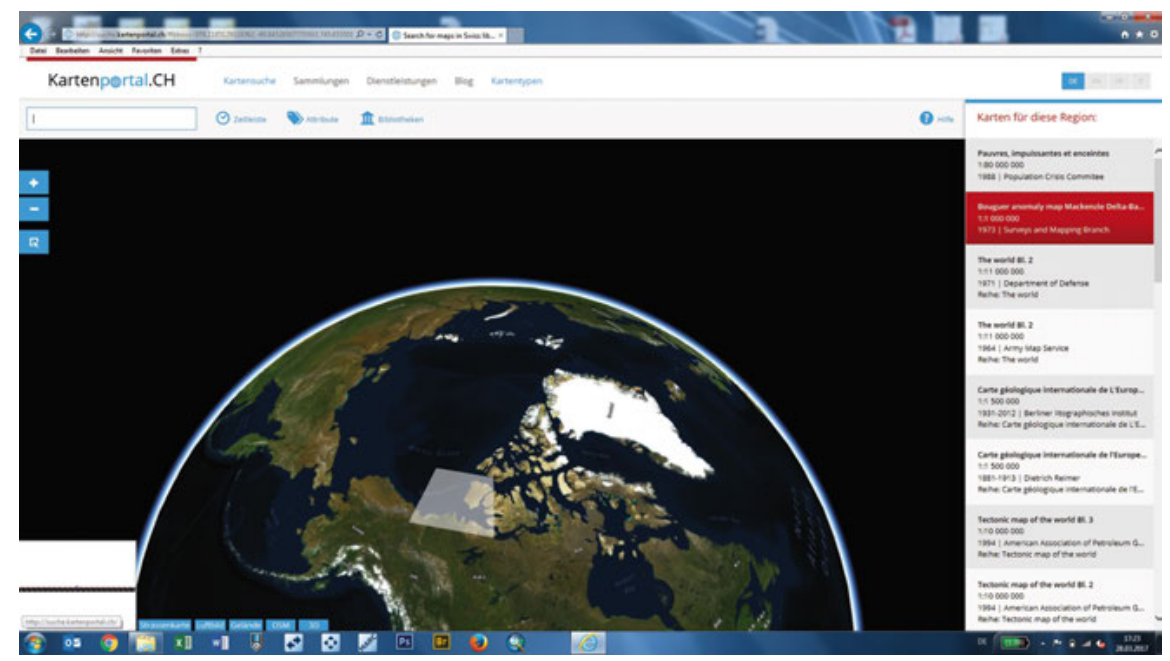

Abb. 4: Seit 2017 ermöglicht ein ,3D‘-Globus als alternative Suchkarte erstmals die räumlichgraphische Recherche nach Karten zu den Polregionen. 
